\title{
Distributed Node-to-Node Consensus of Multiagent Systems with Delayed Nonlinear Dynamics and Intermittent Communications
}

\author{
Kexin Jia, Hongjie Li, Wenqiang Zheng, Qinyou Mou, and Jiajun Shao \\ College of Mathematics and Information and Engineering, Jiaxing University, Zhejiang 314001, China \\ Correspondence should be addressed to Hongjie Li; lhjymlly@163.com
}

Received 13 April 2015; Accepted 2 August 2015

Academic Editor: Yang Tang

Copyright (C) 2015 Kexin Jia et al. This is an open access article distributed under the Creative Commons Attribution License, which permits unrestricted use, distribution, and reproduction in any medium, provided the original work is properly cited.

\begin{abstract}
The paper is concerned with the problem of distributed node-to-node consensus of multiagent systems with delayed nonlinear dynamics and communication constraints. A new kind of consensus protocol based only on the intermittent measurements of neighboring agents is proposed to make each follower track the corresponding leader asymptotically. Based on the Lyapunov stability theory and $M$-matrix theory, some novel and simple criteria are derived for node-to-node consensus of multiagent systems. It is shown that consensus can be reached if the communication time duration is larger than the corresponding threshold value. Finally, a numerical example is provided to demonstrate the effectiveness of the obtained theoretical results.
\end{abstract}

\section{Introduction}

Distributed control of multiagent systems has recently attracted various scientific communities ranging from mathematics to electronic engineering $[1,2]$, which is partly due to its broad applications in sensor networks, formation of unmanned vehicles, clustering of satellites, and so on. An important issue in distributed control of multiagent systems is consensus control, which can be described as only using the local relative information to make the whole system reach a state agreement [3].

Recently, there are many fruitful results on distributed consensus of multiagent systems, according to the presence or absence of a leader in the considered multiagent systems; within the context of multiagent systems, the commonly studied consensus problems can be classified into three categories: leaderless consensus, leader-following, and containment control. Leaderless consensus means that the states of considered multiple agents achieve a prior unknown agreement [4-6]. Leader-following consensus is also called distributed consensus tracking, which will converge to a designed state trajectory which is generated by a virtual or real leader [7-10]. Distributed containment control of multiagent systems with multiagent systems with multiple leaders has been addressed in the literature [11-15], where the control goal is to make the states of each follower converge to a convex combination of the states of all leaders. It should be noted that there is a common assumption in existing works on containment control that there is no interaction among the leaders. However, in some real applications, there do exist interactions between the neighboring leaders and the state of a given follower needs to track that of a particular leader not the convex combination of the states of all leaders. Motivated by this observation, the notion of distributed node-to-node consensus is introduced in $[16,17]$. Specifically, it is assumed that there are two levels in the multiagent systems: the leader's and the follower's levels; the dynamics of each leader may only be affected by those of its neighbors in the leader's levels, while the dynamics of each follower may be affected by those of its neighbors in both leader's and follower's levels. It should be noticed that most of the aforementioned works in multiagent systems are derived on a key assumption that information is transmitted continuously among the agents. However, this assumption may not be ensured in some practical cases due to communication disturbances and sensor range limitations $[18,19]$. Thus it is reasonable to assume that each agent can sense its neighbors only intermittently. It is worth noting that both impulsive 
control and intermittent control are discontinuous controls. The difference of them is that impulsive control is activated only at some isolated points [20-22], while intermittent control is activated during some intervals and does not work during the other intervals. On the other hand, impulsive control and intermittent control have attracted more interest recently due to their easy implementation in engineering control and being more efficient and useful in real-life applications. On the other hand, in order to guarantee stability in complex networks, adaptive strategies to appropriately tune the strengths of the interconnections among network nodes have been proposed [23-25]; it can be proved that the fixed coupling strength is larger than those needed in practice. Therefore, it may be conservative; adaptive strategies are proposed and can effectively overcome these shortcomings, which can appropriately tune the strength with the dynamic evolution of the network. Finally, the characteristic of timedelayed coupling is very common in biological and physical systems; some of the time delays are trivial and so can be ignored, while some others cannot be ignored, such as in long distance communication and traffic congestions. Therefore, time delays should be modeled in order to simulate more realistic networks $[26,27]$.

Motivated by these observations, this paper is devoted to investigate distributed node-to-node consensus problem for a class of multiagent systems with delayed nonlinear dynamics by adaptive intermittent control. The main contributions of this paper can be summarized as follows. (1) The multiagent systems in the present framework consist of two levels, that is, the leader's level and the follower's level. (2) The agent dynamics are extended to delayed nonlinear form. (3) A novel adaptive intermittent control protocol is designed, where only a small fraction of followers can sense their corresponding leaders on some disconnected intervals.

Notation. The notation used here is fairly standard except where otherwise stated. $\mathbb{R}^{n}$ denotes the $n$-dimensional Euclidean space and $\mathbb{R}^{n \times m}$ is a set of real $n \times m$ matrices. $I_{n}$ and $1_{N}$ represent the $n \times n$ identity matrices and the $n$ dimensional column vector with all entries equal to one. $\|\cdot\|$ refers to the Euclidean norm. Let $\operatorname{diag}\{\cdots\}$ stand for a block-diagonal matrix. Symbol $\|\cdot\|$ denotes the Euclidean norm. $M^{T}$ represents the transpose of the matrix $M$. For a real symmetric matrix $A, \lambda_{\max }(A)$ and $\lambda_{\text {min }}(A)$ represent its largest and smallest eigenvalues, respectively. Notation $\otimes$ denotes the Kronecker product. In this paper, if not explicitly stated, matrices are assumed to have compatible dimensions.

\section{Problem Formulation and Preliminaries}

Let $g=(v, \varepsilon, G)$ be a directed graph with the set of nodes $v=\left(v_{1}, v_{2}, \ldots, v_{N}\right)$, the set of directed links $\varepsilon \subseteq v \times v$, and a weighted adjacency matrix $G=\left(G_{i j}\right)_{N \times N}$ with nonnegative adjacency elements $G_{i j}$. A directed graph has or contains a directed spanning tree if there exists a node called root such that there exists a directed path from this node to every other node. The Laplacian matrix $L=\left(l_{i j}\right)_{N \times N}$ of the weighted directed network is defined as $L_{i j}=-G_{i j}(i \neq j) ; L_{i i}=$ $\sum_{j=1, j \neq i}^{N} G_{i j}$
Definition 1. A nonsingular real square matrix $A$ is called an $M$-matrix if all of its off-diagonal elements are nonpositive, and all elements of $A^{-1}$ are nonnegative.

Lemma 2. Suppose that matrix $A \in \mathbb{R}^{n \times n}$ has nonpositive off-diagonal elements; then $A$ is an M-matrix if and only if there exists a positive diagonal matrix $\Xi=$ $\operatorname{diag}\left\{1 / \theta_{1}, 1 / \theta_{2}, \ldots, 1 / \theta_{n}\right\}$, such that $A^{T} \Xi+\Xi A>0$, where $A \theta=1_{N}$ and $\theta=\left[\theta_{1}, \theta_{2}, \ldots, \theta_{n}\right]^{T}$.

Lemma 3. Suppose that nonnegative function $y(t), t \in$ $[-\tau,+\infty)$, satisfies

$$
\dot{y}(t) \leq-c_{1} y(t)+c_{2} y(t-\tau) \quad(t \geq 0) ;
$$

then

$$
y(t) \leq\|y(0)\|_{\tau} e^{-r t} \quad(t \geq 0),
$$

where $c_{1}>c_{2}>0,\|y(0)\|_{\tau}=\max _{-\tau \leq s \leq 0}\{y(s)\}$, and $r$ is the unique solution of $-r=-c_{1}+c_{2} e^{r \tau}$.

Lemma 4. Suppose that nonnegative function $y(t), t \in$ $[-\tau,+\infty)$, satisfies

$$
\dot{y}(t) \leq c_{1} y(t)+c_{2} y(t-\tau) \quad(t \geq 0) ;
$$

then

$$
y(t) \leq\|y(0)\|_{\tau} e^{\left(c_{1}+c_{2}\right) t} \quad(t \geq 0),
$$

where $c_{1}$ and $c_{2}$ are two positive constants and $\|y(0)\|_{\tau}=$ $\max _{-\tau \leq s \leq 0}\{y(s)\}$.

Consider that multiagent systems consist of the leader's and the follower's level; it is assumed that each level contains $N$ coupled agents. The dynamics of the ith agent in the leader's level are described as follows:

$$
\begin{array}{r}
\dot{x}_{i}(t)=f\left(x_{i}(t), x_{i}(t-\tau), t\right)-c \sum_{j=1}^{N} L_{i j} x_{j}(t) \\
\quad(i=1,2, \ldots, N),
\end{array}
$$

where $x_{i}(t) \in \mathbb{R}^{n}$ is the position state of the $i$ th leader. $f(\cdot, \cdot, t): \mathbb{R}^{n} \times \mathbb{R}^{n} \times \mathbb{R}^{+} \rightarrow \mathbb{R}^{n}$ is a continuously differentiable vector-valued function representing the inherent delayed nonlinear dynamics of agent $i . \tau>0$ is the time delay. $c>0$ represents the coupling strength. $L=\left[l_{i j}\right]_{N \times N}$ is the Laplacian matrix of communication topology. Furthermore, the evolution of the $i$ th follower is given as

$$
\begin{array}{r}
\dot{\hat{x}}_{i}(t)=f\left(\widehat{x}_{i}(t), \widehat{x}_{i}(t-\tau), t\right)-c \sum_{j=1}^{N} l_{i j} \widehat{x}_{j}(t)+u_{i}(t) \\
(i=1,2, \ldots, N),
\end{array}
$$

where $\widehat{x}_{i}(t) \in \mathbb{R}^{n}$ is the position state of the $i$ th follower. $u_{i}(t)$ is the control input acting agent $i$.

It is commonly assumed in most existing works that all information is transmitted continuously among the agents. 
However, in some real situations agents may only communicate with their neighbors over some disconnected time intervals due to the unreliability of communication channels, failure of physical devices; thus the adaptive intermittent consensus protocol is designed as follows:

$$
\begin{aligned}
& u_{i}(t) \\
& \quad= \begin{cases}c d_{i}(t)\left(x_{i}(t)-\widehat{x}_{i}(t)\right) & t \in[k T, k T+\delta) \\
0 & t \in[k T+\delta,(k+1) T),\end{cases}
\end{aligned}
$$

where the pinning link $d_{i}(t)>0$ if and only if the $i$ th follower can directly sense the $i$ th leader; otherwise $d_{i}(t)=0$; its updating law is given later. The communication time duration $\delta$ satisfies $\tau<\delta \leq T$.

Remark 5. What kind of agents and how many agents should be pinned? The selection criteria are as follows. Firstly, divide the digraph of the network into $m$ components, where each component contains a directed tree; select the root nodes in these components as pinned nodes. There are two reasons: (1) the related message of the leader can be transmitted to each follower; (2) the consensus can be achieved if and only if the root node of each component is pinned, which means that the minimum number of pinning controllers is $m$. If we select the root nodes in these components as pinned nodes, the following consensus is not achieved; we need to rearrange the remaining nodes in descending order according to the differences of out-degrees and in-degrees and gradually increase the amount of control node, until the consensus is achieved. More detailed discussion can be found in the literatures $[28,29]$.

Definition 6. The distributed node-to-node consensus in multiagent systems (5) and (6) is said to be achieved if, for any initial conditions, the following holds:

$$
\lim _{t \rightarrow \infty}\left\|x_{i}(t)-\widehat{x}_{i}(t)\right\|=0 \quad(i=1,2, \ldots, N) .
$$

\section{Main Result}

In this section, we deal with node-to-node consensus problem for multiagent systems with delayed nonlinear dynamics by adaptive intermittent control. By defining a Lyapunov function and utilizing a combination of stability theory and Kronecker products, some simple conditions are established to ensure node-to-node consensus of multiagent systems (5) and (6). The following assumption is needed to derive the main results.

Assumption 7. For the nonlinear function $f(\cdot, \cdot, t): \mathbb{R}^{n} \times \mathbb{R}^{n} \times$ $\mathbb{R}^{+} \rightarrow \mathbb{R}^{n}$ in (5) and (6), there exist two positive constants $L_{1}, L_{2}>0$ such that

$$
\begin{aligned}
& \left\|f\left(x_{1}, x_{2}, t\right)-f\left(y_{1}, y_{2}, t\right)\right\|^{2} \\
& \quad \leq L_{1}\left\|x_{1}-y_{1}\right\|^{2}+L_{2}\left\|x_{2}-y_{2}\right\|^{2},
\end{aligned}
$$

where $x_{1}, x_{2}, y_{1}, y_{2} \in \mathbb{R}^{n}$.

Substituting (7) into (6), one has

$$
\dot{\hat{x}}_{i}(t)= \begin{cases}f\left(\widehat{x}_{i}(t), \widehat{x}_{i}(t-\tau), t\right)-c \sum_{j=1}^{N} l_{i j} \widehat{x}_{j}(t)+c d_{i}(t)\left(x_{i}(t)-\widehat{x}_{i}(t)\right) & t \in[k T, k T+\delta) \\ f\left(\hat{x}_{i}(t), \widehat{x}_{i}(t-\tau), t\right)-c \sum_{j=1}^{N} l_{i j} \widehat{x}_{j}(t) & t \in[k T+\delta,(k+1) T) .\end{cases}
$$

For convenience, systems (5) and (10) can be rewritten as

$$
\begin{aligned}
& \dot{x}(t)=f(x(t), x(t-\tau), t)-c\left(L \otimes I_{n}\right) x(t), \\
& \dot{\hat{x}}(t)= \begin{cases}f(\widehat{x}(t), \widehat{x}(t-\tau), t)-c\left(L \otimes I_{n}\right) \hat{x}(t)-c\left(D(t) \otimes I_{n}\right)(\widehat{x}(t)-x(t)) & t \in[k T, k T+\delta) \\
f(\widehat{x}(t), \widehat{x}(t-\tau), t)-c\left(L \otimes I_{n}\right) \hat{x}(t) & t \in[k T+\delta,(k+1) T),\end{cases}
\end{aligned}
$$

where

$$
\begin{aligned}
& x(t)=\left[x_{1}^{T}(t), x_{2}^{T}(t), \ldots, x_{N}^{T}(t)\right]^{T}, \\
& \hat{x}(t)=\left[\hat{x}_{1}^{T}(t), \hat{x}_{2}^{T}(t), \ldots, \widehat{x}_{N}^{T}(t)\right]^{T}, \\
& f(x(t), x(t-\tau), t)=\left[f^{T}\left(x_{1}(t), x_{1}(t-\tau), t\right),\right. \\
& f^{T}\left(x_{2}(t), x_{2}(t-\tau), t\right), \ldots,
\end{aligned}
$$

$$
\begin{gathered}
\left.f^{T}\left(x_{N}(t), x_{N}(t-\tau), t\right)\right]^{T}, \\
f(\widehat{x}(t), \widehat{x}(t-\tau), t)=\left[f^{T}\left(\widehat{x}_{1}(t), \widehat{x}_{1}(t-\tau), t\right),\right. \\
f^{T}\left(\widehat{x}_{2}(t), \widehat{x}_{2}(t-\tau), t\right), \ldots, \\
\left.f^{T}\left(\widehat{x}_{N}(t), \widehat{x}_{N}(t-\tau), t\right)\right]^{T}, \\
D(t)=\operatorname{diag}\left\{d_{1}(t), d_{2}(t), \ldots, d_{l}(t), 0, \ldots, 0\right\} .
\end{gathered}
$$


Let $e(t)=\widehat{x}(t)-x(t)$; then the following error system can be obtained from (11) such that

$$
\dot{e}(t)= \begin{cases}f(e(t), e(t-\tau), t)-c\left(L \otimes I_{n}\right) e(t)-c\left(D(t) \otimes I_{n}\right) e(t) & t \in[k T, k T+\delta) \\ f(e(t), e(t-\tau), t)-c\left(L \otimes I_{n}\right) e(t) & t \in[k T+\delta,(k+1) T),\end{cases}
$$

where

$$
\begin{aligned}
f(e(t), e(t-\tau), t)= & f(\widehat{x}(t), \widehat{x}(t-\tau), t) \\
& -f(x(t), x(t-\tau), t) .
\end{aligned}
$$

It is not hard to verify that node-to-node consensus for multiagent systems (5) and (6) will be achieved if and only if zero equilibrium point of error system (13) is globally attractive.

Theorem 8. Suppose that Assumption 7 holds; then distributed node-to-node consensus problem for multiagent systems (5) and (6) is achieved under the following distributed adaptive intermittent control protocol:

$$
u(t)= \begin{cases}-c\left(D(t) \otimes I_{n}\right) e(t) & t \in[k T, k T+\delta) \\ 0 & t \in[k T+\delta,(k+1) T)\end{cases}
$$

and the updating law $d_{i}(t)(i=1,2, \ldots, l)$ is defined as

$$
\begin{aligned}
& \dot{d}_{i}(t) \\
& = \begin{cases}c a_{0 i} e^{a_{1 i} t} e^{T}(t)\left(\Xi \otimes I_{n}\right) e(t) & t \in[k T, k T+\delta) \\
0 & t \in[k T+\delta,(k+1) T)\end{cases}
\end{aligned}
$$

if the following conditions hold:

$$
\begin{aligned}
1+L_{1}-2 c \lambda_{\min }(\bar{L}) \theta_{\min }+a_{1} & <0, \\
1+L_{1}-c \lambda_{\min }(S)-b_{1} & <0, \\
\delta & >\frac{r \tau+\left(b_{1}+L_{2}\right) T}{r+b_{1}+L_{2}},
\end{aligned}
$$

where

$$
\begin{aligned}
& \Xi=\operatorname{diag}\left\{\frac{1}{\theta_{1}}, \frac{1}{\theta_{2}}, \ldots, \frac{1}{\theta_{N}}\right\}, \\
& \theta=\left[\theta_{1}, \theta_{2}, \ldots, \theta_{n}\right]^{T},
\end{aligned}
$$

$$
\begin{aligned}
L \theta & =1_{N}, \\
\theta_{\max } & =\max _{1 \leq i \leq N}\left\{\theta_{i}\right\}, \\
\theta_{\min } & =\min _{1 \leq i \leq N}\left\{\theta_{i}\right\}, \\
\bar{L} & =\frac{\Xi \widehat{L}+\widehat{L}^{T} \Xi}{2}, \\
\widehat{L} & =L+D^{*}, \\
D^{*} & =\operatorname{diag}\left\{d_{1}^{*}, d_{2}^{*}, \ldots, d_{l}^{*}, 0, \ldots, 0\right\}, \\
S & =\Xi^{-1}\left(\Xi L+L^{T} \Xi\right), \\
-r & =-a_{1}+L_{2} e^{r \tau}, \\
a_{1} & =\min _{1 \leq i \leq N}\left\{a_{1 i}\right\} .
\end{aligned}
$$

Proof. Choose the following Lyapunov function candidate:

$$
\begin{aligned}
V(t)= & \frac{1}{2} e^{T}(t)\left(\Xi \otimes I_{n}\right) e(t) \\
& +\frac{1}{2} \sum_{i=1}^{l} e^{-a_{1 i} t} \frac{\left(d_{i}(t)-d^{*}\right)^{2}}{a_{0 i}},
\end{aligned}
$$

where $\Xi=\operatorname{diag}\left\{1 / \theta_{1}, 1 / \theta_{2}, \ldots, 1 / \theta_{N}\right\}, \theta=\left[\theta_{1}, \theta_{2}, \ldots, \theta_{n}\right]^{T}$ satisfies $L \theta=1_{N} \cdot d_{i}^{*}(i=1,2, \ldots, l)$ are some undetermined sufficiently large positive constants.

(1) For $t \in[k T, k T+\delta)$ and an arbitrarily given $k \in \mathbb{N}$, taking the derivative of $V(t)$ along the trajectories of system (13) can be given as follows:

$$
\begin{aligned}
& \dot{V}(t)=e^{T}(t)\left(\Xi \otimes I_{n}\right) \dot{e}(t)-\frac{1}{2} \\
& \cdot \sum_{i=1}^{l} a_{1 i} e^{-a_{1 i} t} \frac{\left(d_{i}(t)-d_{i}^{*}\right)^{2}}{a_{0 i}} \\
& +\sum_{i=1}^{l} e^{-a_{1 i} t} \frac{\left(d_{i}(t)-d_{i}^{*}\right)}{a_{0 i}} \dot{d}_{i}(t)=e^{T}(t)\left(\Xi \otimes I_{n}\right) \\
& \cdot f(e(t), e(t-\tau), t)-c e^{T}(t)\left(\frac{\Xi L+L^{T} \Xi}{2} \otimes I_{n}\right) \\
& \cdot e(t)-c e^{T}(t)\left(\frac{\Xi D(t)+D(t)^{T} \Xi}{2} \otimes I_{n}\right) e(t)-\frac{1}{2}
\end{aligned}
$$




$$
\begin{aligned}
& \cdot \sum_{i=1}^{l} a_{1 i} e^{-a_{1 i} t} \frac{\left(d_{i}(t)-d_{i}^{*}\right)^{2}}{a_{0 i}} \\
& +\sum_{i=1}^{l}\left(d_{i}(t)-d_{i}^{*}\right) e^{T}(t)\left(\Xi \otimes I_{n}\right) \dot{e}(t)=e^{T}(t) \\
& \cdot\left(\Xi \otimes I_{n}\right) f(e(t), e(t-\tau), t)-c e^{T}(t)\left(\bar{L} \otimes I_{n}\right) \\
& \cdot e(t)-\frac{1}{2} \sum_{i=1}^{l} a_{1 i} e^{-a_{1 i} t} \frac{\left(d_{i}(t)-d_{i}^{*}\right)^{2}}{a_{0 i}} \leq \frac{1}{2} e^{T}(t) \\
& \cdot\left(\Xi \otimes I_{n}\right) e(t)+\frac{1}{2} f^{T}(e(t), e(t-\tau), t)\left(\Xi \otimes I_{n}\right) \\
& \cdot f(e(t), e(t-\tau), t)-c e^{T}(t)\left(\bar{L} \otimes I_{n}\right) e(t)-\frac{1}{2} \\
& \cdot \sum_{i=1}^{l} a_{1 i} e^{-a_{1 i} t} \frac{\left(d_{i}(t)-d_{i}^{*}\right)^{2}}{a_{0 i}} \leq \frac{1}{2} e^{T}(t)\left(\Xi \otimes I_{n}\right) e(t) \\
& +\frac{1}{2} L_{1} e^{T}(t)\left(\Xi \otimes I_{n}\right) e(t)+\frac{1}{2} L_{2} e^{T}(t-\tau)\left(\Xi \otimes I_{n}\right) \\
& \cdot e(t-\tau)-c \lambda_{\min }(\bar{L}) \theta_{\min } e^{T}(t)\left(\Xi \otimes I_{n}\right) e(t)-\frac{1}{2} \\
& \cdot \sum_{i=1}^{l} a_{1 i} e^{-a_{1 i} t} \frac{\left(d_{i}(t)-d_{i}^{*}\right)^{2}}{a_{0 i}} \\
& =\frac{1}{2}\left(1+L_{1}-2 c \lambda_{\min }(\bar{L}) \theta_{\min }\right) e^{T}(t)\left(\Xi \otimes I_{n}\right) e(t) \\
& -\frac{1}{2} \sum_{i=1}^{l} a_{1 i} e^{-a_{1 i} t} \frac{\left(d_{i}(t)-d_{i}^{*}\right)^{2}}{a_{0 i}}+\frac{1}{2} L_{2} e^{T}(t-\tau) \\
& \cdot\left(\Xi \otimes I_{n}\right) e(t-\tau) \\
& \leq \frac{1}{2}\left(1+L_{1}-2 c \lambda_{\min }(\bar{L}) \theta_{\min }+a_{1}\right) e^{T}(t)\left(\Xi \otimes I_{n}\right) \\
& \cdot e(t)-\frac{1}{2} a_{1} e^{T}(t)\left(\Xi \otimes I_{n}\right) e(t)-\frac{1}{2} \\
& \cdot a_{1} \sum_{i=1}^{l} e^{-a_{1 i} t} \frac{\left(d_{i}(t)-d_{i}^{*}\right)^{2}}{a_{0 i}}+\frac{1}{2} L_{2} e^{T}(t-\tau)\left(\Xi \otimes I_{n}\right) \\
& \cdot e(t-\tau)+\frac{1}{2} L_{2} \sum_{i=1}^{l} e^{-a_{1 i}(t-\tau)} \frac{\left(d_{i}(t-\tau)-d_{i}^{*}\right)^{2}}{a_{0 i}} \\
& \leq-a_{1} V(t)+L_{2} V(t-\tau)
\end{aligned}
$$

where

$$
\begin{aligned}
& a_{1}=\min _{1 \leq i \leq N}\left\{a_{1 i}\right\}, \\
& \bar{L}=\frac{\Xi \widehat{L}+\widehat{L}^{T} \Xi}{2},
\end{aligned}
$$

$$
\begin{aligned}
\widehat{L} & =L+D^{*}, \\
D^{*} & =\operatorname{diag}\left\{d_{1}^{*}, d_{2}^{*}, \ldots, d_{l}^{*}, 0, \ldots, 0\right\}, \\
\theta_{\min } & =\min _{1 \leq i \leq N}\left\{\theta_{i}\right\} .
\end{aligned}
$$

(2) For $t \in[k T+\delta,(k+1) T)$ with arbitrarily given $k \in \mathbb{N}$, taking the time derivative $V(t)$ along the trajectories of (13), we have

$$
\begin{aligned}
& \dot{V}(t)=e^{T}(t)\left(\Xi \otimes I_{n}\right) \dot{e}(t)-\frac{1}{2} \\
& \cdot \sum_{i=1}^{l} a_{1 i} e^{-a_{1 i} t} \frac{\left(d_{i}(t)-d_{i}^{*}\right)^{2}}{a_{0 i}}=e^{T}(t)\left(\Xi \otimes I_{n}\right) \\
& \cdot f(e(t), e(t-\tau), t)-c e^{T}(t)\left(\widetilde{L} \otimes I_{n}\right) e(t)-\frac{1}{2} \\
& \cdot \sum_{i=1}^{l} a_{1 i} e^{-a_{1 i} t} \frac{\left(d_{i}(t)-d_{i}^{*}\right)^{2}}{a_{0 i}} \leq \frac{1}{2} e^{T}(t)\left(\Xi \otimes I_{n}\right) e(t) \\
& +\frac{1}{2} f^{T}(e(t), e(t-\tau), t)\left(\Xi \otimes I_{n}\right) \\
& \cdot f(e(t), e(t-\tau), t)-c e^{T}(t)\left(\widetilde{L} \otimes I_{n}\right) e(t)-\frac{1}{2} \\
& \cdot \sum_{i=1}^{l} a_{1 i} e^{-a_{1 i} t} \frac{\left(d_{i}(t)-d_{i}^{*}\right)^{2}}{a_{0 i}} \leq \frac{1}{2}\left(1+L_{1}\right) e^{T}(t) \\
& \cdot\left(\Xi \otimes I_{n}\right) e(t)+\frac{1}{2} L_{2} e^{T}(t-\tau)\left(\Xi \otimes I_{n}\right) e(t-\tau) \\
& -c e^{T}(t)\left(\widetilde{L} \otimes I_{n}\right) e(t)-\frac{1}{2} \sum_{i=1}^{l} a_{1 i} e^{-a_{1 i} t} \frac{\left(d_{i}(t)-d_{i}^{*}\right)^{2}}{a_{0 i}}
\end{aligned}
$$

where $\widetilde{L}=\left(\Xi L+L^{T} \Xi\right) / 2$.

Note that, for an arbitrarily given $t \in[k T+\delta,(k+1) T)$, the matrix $\left\langle\Xi L+L^{T} \Xi, \Xi\right\rangle$ is normal; for any given $z \in \mathbb{R}^{N}$, it is easily derived from the generalized eigenvalue theory that

$$
\lambda_{\min }(S) z^{T} \Xi z \leq z^{T}\left(\Xi L+L^{T} \Xi\right) z
$$

where $S=\Xi^{-1}\left(\Xi L+L^{T} \Xi\right)$; then one has

$$
\frac{1}{2} \lambda_{\min }(S) e^{T}(t)\left(\Xi \otimes I_{n}\right) e(t) \leq e^{T}(t)\left(\widetilde{L} \otimes I_{n}\right) e(t)
$$

By considering (18), (24), and (26), we can obtain

$\dot{V}(t)$

$$
\begin{aligned}
\leq & \frac{1}{2}\left(1+L_{1}\right) e^{T}(t)\left(\Xi \otimes I_{n}\right) e(t) \\
& +\frac{1}{2} L_{2} e^{T}(t-\tau)\left(\Xi \otimes I_{n}\right) e(t-\tau)
\end{aligned}
$$




$$
\begin{aligned}
& -\frac{1}{2} c \lambda_{\min }(S) e^{T}(t)\left(\Xi \otimes I_{n}\right) e(t) \\
& -\frac{1}{2} \sum_{i=1}^{l} a_{1 i} e^{-a_{1 i} t} \frac{\left(d_{i}(t)-d_{i}^{*}\right)^{2}}{a_{0 i}} \\
\leq & \frac{1}{2}\left(1+L_{1}-c \lambda_{\min }(S)-b_{1}\right) e^{T}(t)\left(\Xi \otimes I_{n}\right) e(t) \\
+ & \frac{1}{2} b_{1} e^{T}(t)\left(\Xi \otimes I_{n}\right) e(t) \\
& +\frac{1}{2} b_{1} \sum_{i=1}^{l} e^{-a_{1 i} t} \frac{\left(d_{i}(t)-d_{i}^{*}\right)^{2}}{a_{0 i}} \\
& +\frac{1}{2} L_{2} e^{T}(t-\tau)\left(\Xi \otimes I_{n}\right) e(t-\tau) \\
& +\frac{1}{2} L_{2} \sum_{i=1}^{l} e^{-a_{1 i}(t-\tau)} \frac{\left(d_{i}(t-\tau)-d_{i}^{*}\right)^{2}}{a_{0 i}} \\
\leq & b_{1} V(t)+L_{2} V(t-\tau),
\end{aligned}
$$

where $b_{1}>0$.

Based on the above analysis and according to Lemma 3, when $t \in[0, \delta)$, we can obtain

$$
V(t) \leq \max _{-\tau \leq s \leq 0} V(s) e^{-r t}=\|V(0)\|_{\tau} e^{-r t}
$$

where $r$ is the positive solution of $-r=-a_{1}+L_{2} e^{-r \tau}$.

When $t \in[\delta, T)$, by using Lemma 4 , one has

$$
\begin{aligned}
V(t) & \leq \max _{\delta-\tau \leq s \leq \delta} V(s) e^{\left(b_{1}+L_{2}\right)(t-\delta)} \\
& \leq \max _{-\tau \leq s \leq 0} V(s)\left\{\max _{\delta-\tau \leq t \leq \delta} e^{-r t}\right\} e^{\left(b_{1}+L_{2}\right)(t-\delta)} \\
& =\|V(0)\|_{\tau} e^{-r(\delta-\tau)+\left(b_{1}+L_{2}\right)(t-\delta)}
\end{aligned}
$$

Consider $T-\tau>\delta$; then

$$
\begin{aligned}
\|V(t)\|_{\tau} & =\max _{T-\tau \leq t \leq T} V(t) \\
& \leq \max _{\delta-\tau \leq s \leq \delta} V(s) \max _{T-\tau \leq t \leq T}\left\{e^{\left(b_{1}+L_{2}\right)(t-\delta)}\right\} \\
& \leq \max _{\delta-\tau \leq s \leq \delta} V(s) e^{\left(b_{1}+L_{2}\right)(T-\delta)} \\
& \leq \max _{-\tau \leq s \leq 0} V(s) e^{-r(\delta-\tau)+\left(b_{1}+L_{2}\right)(T-\delta)} \\
& =\|V(0)\|_{\tau} e^{-\Delta},
\end{aligned}
$$

where $\Delta=r(\delta-\tau)-\left(b_{1}+L_{2}\right)(T-\delta)$.
Next, for all $k \in \mathbb{N}$, we will prove that $\|V(k T)\|_{\tau} \leq$ $\|V(0)\|_{\tau} e^{-k \Delta}$. One has

$$
\begin{aligned}
& \|V(k T)\|_{\tau}=\max _{k T-\tau \leq t \leq k T} V(t) \\
& \leq \max _{(k-1) T+\delta-\tau \leq s \leq(k-1) T+\delta} V(s) \\
& \cdot \max _{k T-\tau \leq t \leq k T} e^{\left(b_{1}+L_{2}\right)(t-(k-1) T-\delta)} \\
& =\max _{(k-1) T+\delta-\tau \leq s \leq(k-1) T+\delta} V(s) e^{\left(b_{1}+L_{2}\right)(T-\delta)} \\
& \leq \max _{(k-1) T-\tau \leq s \leq(k-1) T} V(s) \\
& \cdot\left\{\max _{(k-1) T+\delta-\tau \leq t \leq(k-1) T+\delta} e^{-r(t-(k-1) T)}\right\} e^{\left(b_{1}+L_{2}\right)(T-\delta)} \\
& =\max _{(k-1) T-\tau \leq s \leq(k-1) T} V(s) e^{-r(\delta-\tau)+\left(b_{1}+L_{2}\right)(T-\delta)} \\
& =\max _{(k-1) T-\tau \leq s \leq(k-1) T} V(s) e^{-\Delta}=\cdots \leq \max _{-\tau \leq s \leq 0} V(s) e^{-k \Delta} \\
& =\|V(0)\|_{\tau} e^{-k \Delta} .
\end{aligned}
$$

For any $t>0$, there exists a positive integer $k_{0}$, such that $k_{0} T \leq t \leq\left(k_{0}+1\right) T$; then one has the following.

When $t \in\left[k_{0} T, k_{0} T+\delta\right)$, we can obtain

$$
\begin{aligned}
V(t) & \leq \max _{k_{0} T-\tau \leq s \leq k_{0} T} V(s) e^{-r\left(t-k_{0} T\right)} \leq \max _{-\tau \leq s \leq 0} V(s) e^{-k_{0} \Delta} \\
& \leq \max _{-\tau \leq s \leq 0} V(s) e^{\Delta(1-t / T)}=\|V(0)\|_{\tau} e^{\Delta} e^{-(\Delta / T) t} .
\end{aligned}
$$

When $t \in\left[k_{0} T+\delta,\left(k_{0}+1\right) T\right)$, we have

$$
\begin{aligned}
& V(t) \leq \max _{k_{0} T+\delta-\tau \leq s \leq k_{0} T+\delta} V(s) e^{\left(b_{1}+L_{2}\right)\left(t-k_{0} T-\delta\right)} \\
& \leq \max _{k_{0} T-\tau \leq s \leq k_{0} T} V(s)\left\{\max _{k_{0} T+\delta-\tau \leq t \leq k_{0} T+\delta} e^{-r\left(t-k_{0} T\right)}\right\} \\
& \cdot e^{\left(b_{1}+L_{2}\right)\left(t-k_{0} T-\delta\right)} \\
& =\max _{k_{0} T-\tau \leq s \leq k_{0} T} V(s) e^{-r(\delta-\tau)} e^{\left(t-\left(k_{0}+1\right) T\right)+T-\delta} \\
& \leq\|V(0)\|_{\tau} e^{-k_{0} \Delta} e^{-r(\delta-\tau)+\left(b_{1}+L_{2}\right)(T-\delta)}=\|V(0)\|_{\tau} \\
& \cdot e^{-\left(k_{0}+1\right) \Delta} \leq\|V(0)\|_{\tau} e^{-(\Delta / T) t} .
\end{aligned}
$$

From (32) and (33), we can obtain

$$
V(t) \leq K_{0} e^{-(\Delta / T) t}
$$




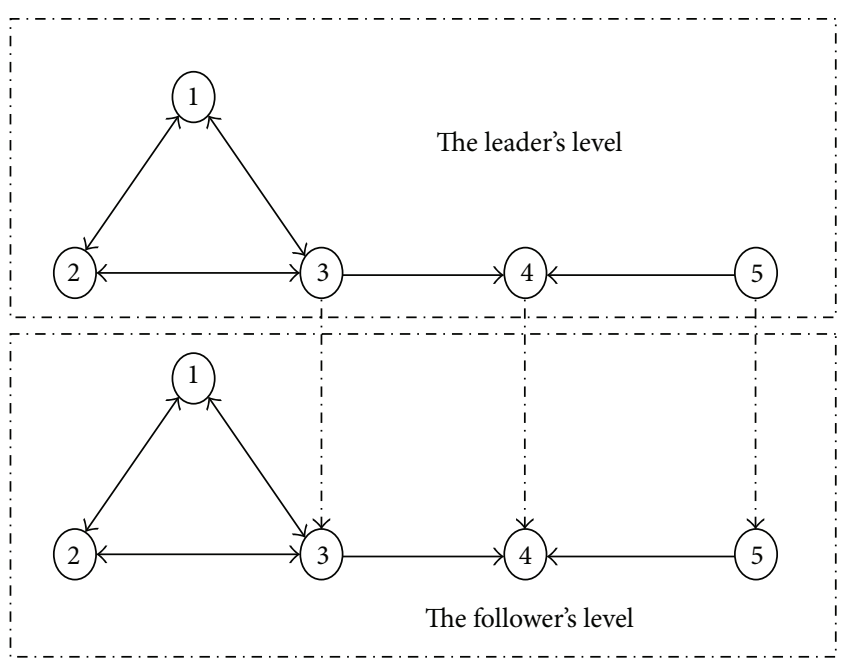

FIGURE 1: Communication topology of multiagent systems (5) and (6).

where $K_{0}=\max \left\{1, e^{\Delta}\right\}$, which indicates that the states of agents exponentially converge to node-to-node consensus. This completes the proof.

Remark 9. It can be seen from (34) that the convergence speed is related to the value of $\Delta$; by some simple computation, we have

$$
\begin{aligned}
\Delta & =r \delta-r \tau-\left(b_{1}+L_{2}\right) T+\left(b_{1}+L_{2}\right) \delta \\
& =\left(r+b_{1}+L_{2}\right) \delta-r \tau-\left(b_{1}+L_{2}\right) T ;
\end{aligned}
$$

from the above equation, we can see that the convergence speed will accelerate with the increase of $\delta$. Therefore, a tradeoff has to be made between the convergence rate and the control cost.

\section{A Numerical Example}

In this section, a numerical example is provided to verify the analysis results.

Example 1. Suppose that there are five leaders in the considered multiagent systems; the communication topologies among the leaders and the followers are given in Figure 1; the solid lines indicate the neighboring relationship among the agents in the same level, while the dashed lines are the pinning links; the corresponding Laplacian matrix $L$ and the pinning matrix $D^{*}$ are given as follows:

$$
L=\left[\begin{array}{ccccc}
4 & -3 & -1 & 0 & 0 \\
-3 & 5 & -2 & 0 & 0 \\
-1 & -2 & 3 & 0 & 0 \\
0 & 0 & -2 & 3 & -1 \\
0 & 0 & 0 & 0 & 0
\end{array}\right]
$$

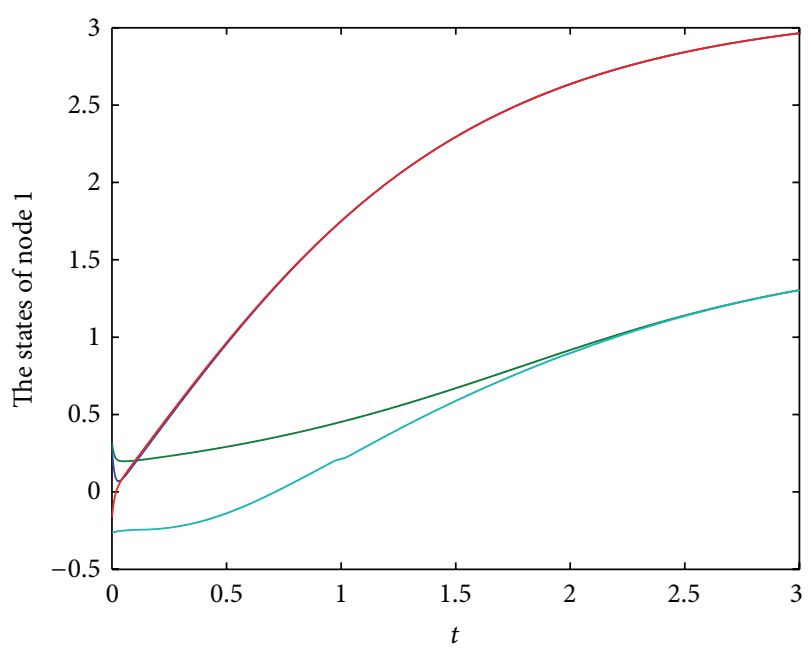

Figure 2: The curves of position states $x_{1}(t)$ and $\widehat{x}_{1}(t)$.

$$
D^{*}=\left[\begin{array}{ccccc}
0 & 0 & 0 & 0 & 0 \\
0 & 0 & 0 & 0 & 0 \\
0 & 0 & 10 & 0 & 0 \\
0 & 0 & 0 & 1 & 0 \\
0 & 0 & 0 & 0 & 1
\end{array}\right] .
$$

The nonlinear dynamics are described as $f\left(x_{i}(t), x_{i}(t-\right.$ $\tau), t)=\left[\cos \left(0.5 x_{i 1}(t)\right)+\cos \left(0.5 x_{i 1}(t-\tau)\right), 0.25 \sin \left(2 x_{i 2}(t)\right)+\right.$ $\left.0.25 \sin \left(2 x_{i 2}(t-\tau)\right)\right]^{T}$; it can be verified that the intrinsic nonlinear dynamics of the agents obey Assumption 7 with $L_{1}=L_{2}=0.5$.

By some simple computation, we have

$$
\begin{aligned}
& \theta=\left[\begin{array}{lllll}
1.0273 & 0.9364 & 0.3 & 0.1308 & 0.1
\end{array}\right], \\
& \Xi=\operatorname{diag}\left\{\begin{array}{lllll}
0.9735 & 1.068 & 3.3333 & 7.6471 & 10
\end{array}\right\} ;
\end{aligned}
$$

then we can obtain $\theta_{\min }=0.1, \lambda_{\min }(\bar{L})=0.8816$, and $\lambda_{\text {min }}(S)=-0.8499$. Taking $c=11.4, T=1$, and $\tau=$ 0.1 , by using condition (17) in Theorem 8 , we can obtain $a_{1}<0.51$; let $a_{1}=0.5099$. By using condition (18), we have $b_{1}>11.1319$, here taking $b_{1}=11.14$. Then we can obtain the communication $\delta>0.9646$, taking $\delta=0.9650$. It is assumed that the agents communicate with their neighbors only when $t \in \bigcup_{k \in N}[k, k+0.9650)$, which indicates that the average communication rate is 0.9650 . The position state trajectories of the leaders and the followers are shown in Figures 2-6, which indicate that node-to-node consensus in multiagent systems (5) and (6) is indeed guaranteed. The position state error trajectories of agents are shown in Figure 7, which further demonstrate the effectiveness of the obtained criteria. The curve of adaptive intermittent control is shown in Figure 8; it is noted that the control $u(t)=0$ in time interval $\bigcup_{k \in N}[k+0.9650, k+1)$. The trajectory of pinning feedback gain $d_{i}(t)(i=3,4,5)$ under the updating 


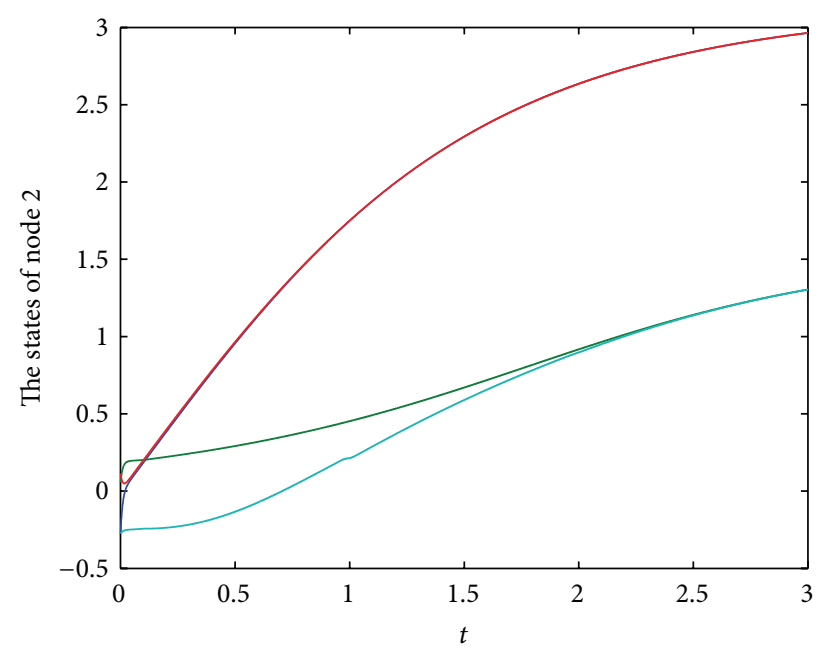

Figure 3: The curves of position states $x_{2}(t)$ and $\widehat{x}_{2}(t)$.

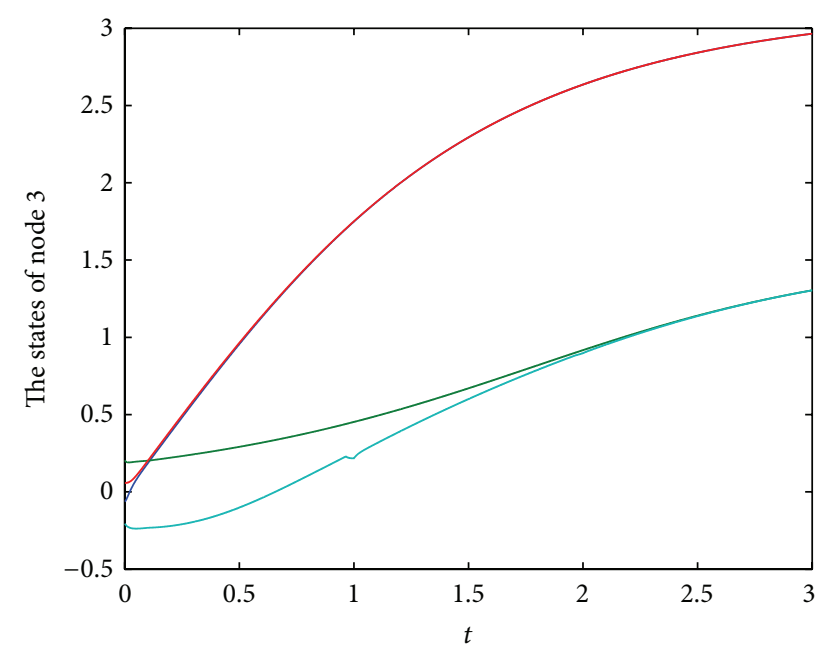

Figure 4: The curves of position states $x_{3}(t)$ and $\widehat{x}_{3}(t)$.

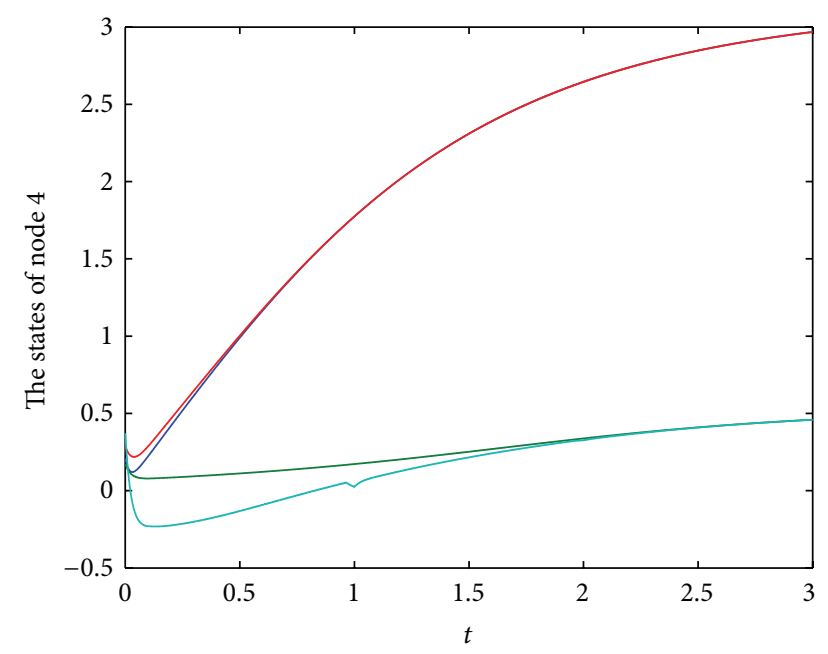

Figure 5: The curves of position states $x_{4}(t)$ and $\widehat{x}_{4}(t)$.

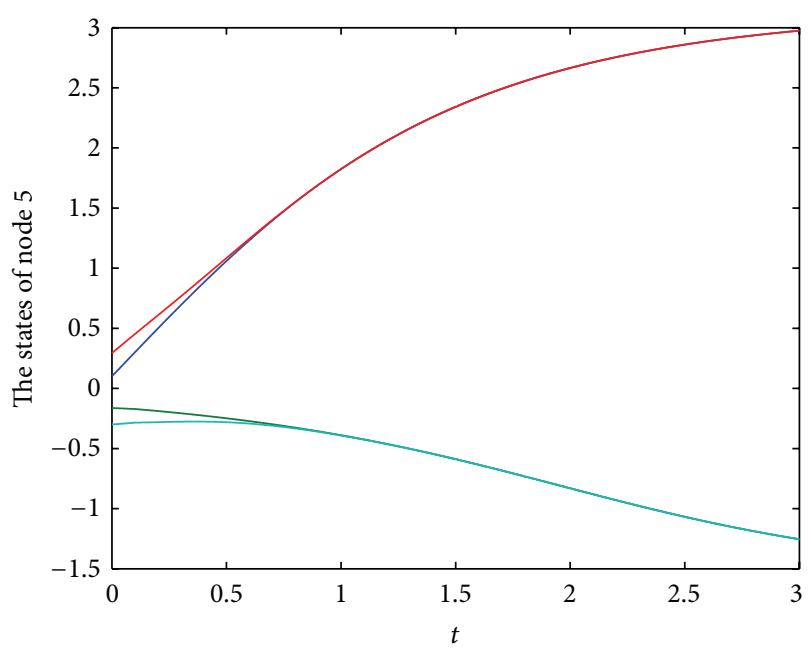

FIgURE 6: The curves of position states $x_{5}(t)$ and $\widehat{x}_{5}(t)$.

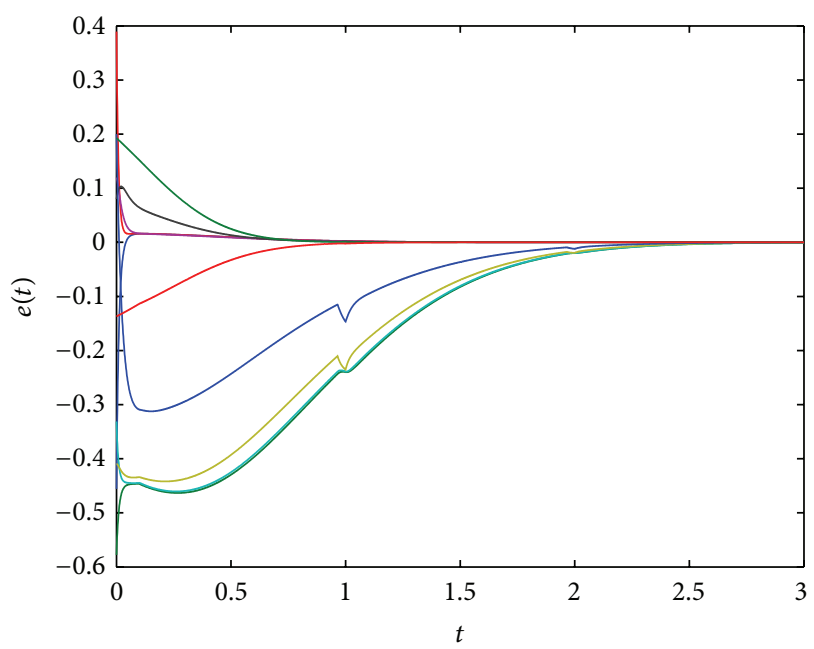

FIGURE 7: The position state error trajectories of $e(t)$.

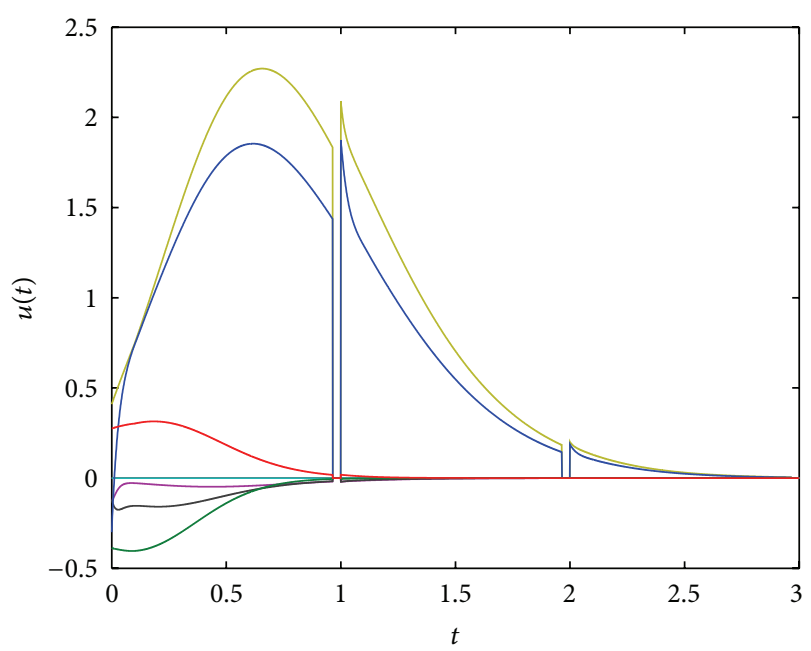

FIGURE 8: The trajectory of adaptive intermit control $u(t)$. 


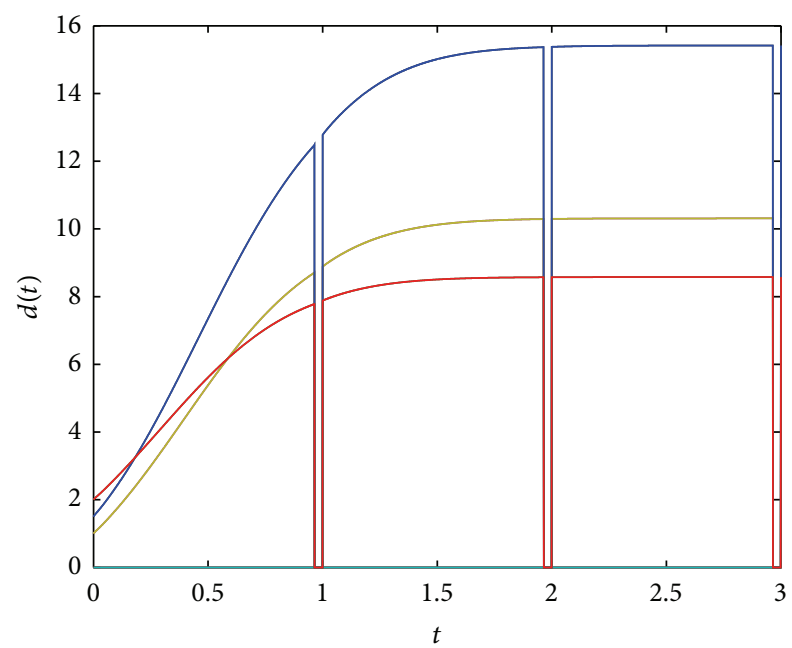

Figure 9: The trajectory of pinning feedback gains $d_{i}(t)(i=3,4,5)$.

laws (16) is shown in Figure 9. The simulation has confirmed the effectiveness of our theoretical results.

\section{Conclusions}

In this paper, we have investigated node-to-node consensus of multiagent systems with delayed nonlinear dynamics. The coordination goal is to make each follower track the corresponding leader asymptotically, where only a small fraction of followers can sense the states of their corresponding leaders on some disconnected time intervals. Finally, a simulation example has been employed to demonstrate the effectiveness of the results. Further research topics include the extension of our results to second-order or high-order multiagent systems with delayed nonlinear dynamics and communication constraints.

\section{Conflict of Interests}

The authors declare that there is no conflict of interests regarding the publication of this paper.

\section{Acknowledgments}

This work was jointly supported by National Natural Science Foundation of China under Grant nos. 61272034 and 11301226 and Zhejiang Provincial Natural Science Foundation of China under Grant nos. LY13F030012 and LQ13A010017.

\section{References}

[1] R. Olfati-Saber, J. A. Fax, and R. M. Murray, "Consensus and cooperation in networked multi-agent systems," Proceedings of the IEEE, vol. 95, no. 1, pp. 215-233, 2007.

[2] W. Ren, R. W. Beard, and E. M. Atkins, "Information consensus in multivehicle cooperative control," IEEE Control Systems, vol. 27, no. 2, pp. 71-82, 2007.

[3] R. Olfati-Saber and R. M. Murray, "Consensus problems in networks of agents with switching topology and time-delays,"
IEEE Transactions on Automatic Control, vol. 49, no. 9, pp. 15201533,2004

[4] J. H. Lu, "Synchronization of complex networks and consensus of complex multi-agent systems," Complex Systems and Complexity Science, vol. 7, no. 2-3, pp. 50-52, 2010.

[5] P. Lin, Y. Jia, and L. Li, "Distributed robust $H_{\infty}$ consensus control in directed networks of agents with time-delay," Systems \& Control Letters, vol. 57, no. 8, pp. 643-653, 2008.

[6] W. Yu, G. Chen, W. Ren, J. Kurths, and W. X. Zheng, "Distributed higher order consensus protocols in multiagent dynamical systems," IEEE Transactions on Circuits and Systems I: Regular Papers, vol. 58, no. 8, pp. 1924-1932, 2011.

[7] W. Ni and D. Cheng, "Leader-following consensus of multiagent systems under fixed and switching topologies," Systems and Control Letters, vol. 59, no. 3-4, pp. 209-217, 2010.

[8] Q. Song, J. Cao, and W. Yu, "Second-order leader-following consensus of nonlinear multi-agent systems via pinning control," Systems \& Control Letters, vol. 59, no. 9, pp. 553-562, 2010.

[9] L. Ding, Q.-L. Han, and G. Guo, "Network-based leaderfollowing consensus for distributed multi-agent systems," Automatica, vol. 49, no. 7, pp. 2281-2286, 2013.

[10] Z.-H. Guan, F.-L. Sun, Y.-W. Wang, and T. Li, "Finite-time consensus for leader-following second-order multi-agent networks," IEEE Transactions on Circuits and Systems I: Regular Papers, vol. 59, no. 11, pp. 2646-2654, 2012.

[11] M. Ji, G. Ferrari-Trecate, M. Egerstedt, and A. Buffa, "Containment control in mobile networks," IEEE Transactions on Automatic Control, vol. 53, no. 8, pp. 1972-1975, 2008.

[12] Z. Meng, W. Ren, and Z. You, "Distributed finite-time attitude containment control for multiple rigid bodies," Automatica, vol. 46, no. 12, pp. 2092-2099, 2010.

[13] Y. Lou and Y. Hong, "Target containment control of multi-agent systems with random switching interconnection topologies," Automatica, vol. 48, no. 5, pp. 879-885, 2012.

[14] S. Liu, L. Xie, and H. Zhang, "Containment control of multiagent systems by exploiting the control inputs of neighbors," International Journal of Robust and Nonlinear Control, vol. 24, no. 17, pp. 2803-2818, 2014.

[15] Y. Zheng and L. Wang, "Containment control of heterogeneous multi-agent systems," International Journal of Control, vol. 87, no. 1, pp. 1-8, 2014.

[16] G. Wen, W. Yu, Y. Zhao, and J. Cao, "Node-to-node consensus of networked agents with general linear node dynamics," in Proceedings of the 3rd Annual IEEE International Conference on Cyber Technology in Automation, Control, and Intelligent Systems (CYBER '13), pp. 24-29, Nanjing, China, May 2013.

[17] G. Wen, W. Yu, J. Wang, D. Xu, and J. Cao, "Node-to-node consensus of multi-agent systems with switched pinning links," in Proceedings of the 26th Chinese Control and Decision Conference (CCDC '14), pp. 269-273, Changsha, China, June 2014.

[18] G. Wen, Z. Duan, Z. Li, and G. Chen, "Consensus and its 12-gain performance of multi-agent systems with intermittent information transmissions," International Journal of Control, vol. 85, no. 4, pp. 384-396, 2012.

[19] G. Wen, Z. Duan, W. Yu, and G. Chen, "Consensus of secondorder multi-agent systems with delayed nonlinear dynamics and intermittent communications," International Journal of Control, vol. 86, no. 2, pp. 322-331, 2013.

[20] Z.-H. Guan, Z.-W. Liu, G. Feng, and Y.-W. Wang, "Synchronization of complex dynamical networks with time-varying delays via impulsive distributed control," IEEE Transactions on Circuits and Systems I: Regular Papers, vol. 57, no. 8, pp. 2182-2195, 2010. 
[21] Z.-W. Liu, Z.-H. Guan, X. M. Shen, and G. Feng, "Consensus of multi-agent networks with aperiodic sampled communication via impulsive algorithms using position-only measurements," IEEE Transactions on Automatic Control, vol. 57, no. 10, pp. 2639-2643, 2012.

[22] Y. Tang, H. J. Gao, W. B. Zhang, and J. Kurths, "Leader-following consensus of a class of stochastic delayed multi-agent systems with partial mixed impulses," Automatica, vol. 53, pp. 346-354, 2015.

[23] W. Yu, P. DeLellis, G. Chen, M. di Bernardo, and J. Kurths, "Distributed adaptive control of synchronization in complex networks," IEEE Transactions on Automatic Control, vol. 57, no. 8, pp. 2153-2158, 2012.

[24] Z. Li, L. Jiao, and J.-J. Lee, "Robust adaptive global synchronization of complex dynamical networks by adjusting timevarying coupling strength," Physica A: Statistical Mechanics and its Applications, vol. 387, no. 5-6, pp. 1369-1380, 2008.

[25] L. Huang, Z. Wang, Y. Wang, and Y. Zuo, "Synchronization analysis of delayed complex networks via adaptive time-varying coupling strengths," Physics Letters A, vol. 373, no. 43, pp. 39523958, 2009.

[26] R. Lu, H. Wu, and J. Bai, "New delay-dependent robust stability criteria for uncertain neutral systems with mixed delays," Journal of the Franklin Institute, vol. 351, no. 3, pp. 1386-1399, 2014.

[27] R. Q. Lu, H. Y. Su, J. Chu, and A. K. Xue, "A simple approach to robust $D$-stability analysis for uncertain singular delay systems," Asian Journal of Control, vol. 11, no. 4, pp. 411-419, 2009.

[28] Q. Song, F. Liu, J. Cao, and W. Yu, "Pinning-controllability analysis of complex networks: an M-matrix approach," IEEE Transactions on Circuits and Systems I: Regular Papers, vol. 59, no. 11, pp. 2692-2701, 2012.

[29] Q. Song, J. Cao, and W. Yu, "Second-order leader-following consensus of nonlinear multi-agent systems via pinning control," Systems and Control Letters, vol. 59, no. 9, pp. 553-562, 2010. 


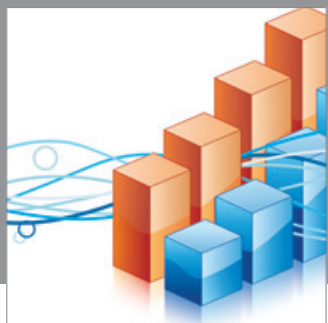

Advances in

Operations Research

mansans

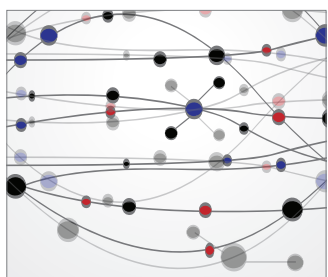

The Scientific World Journal
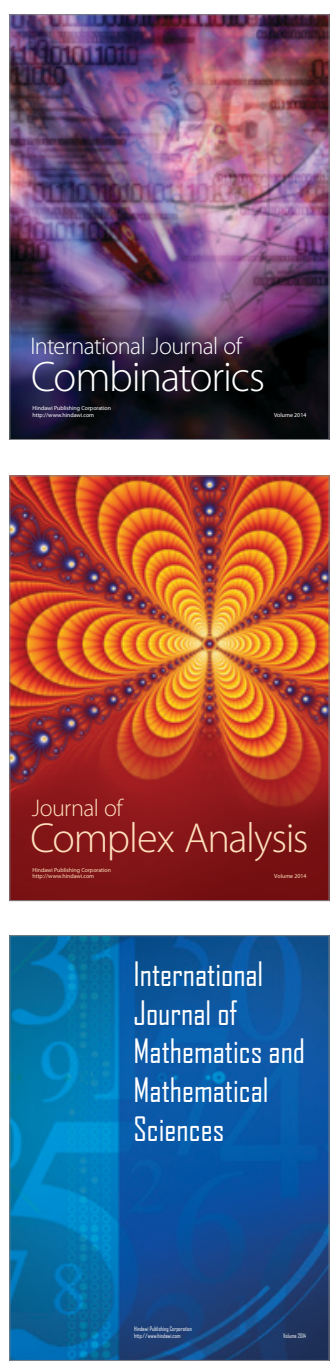
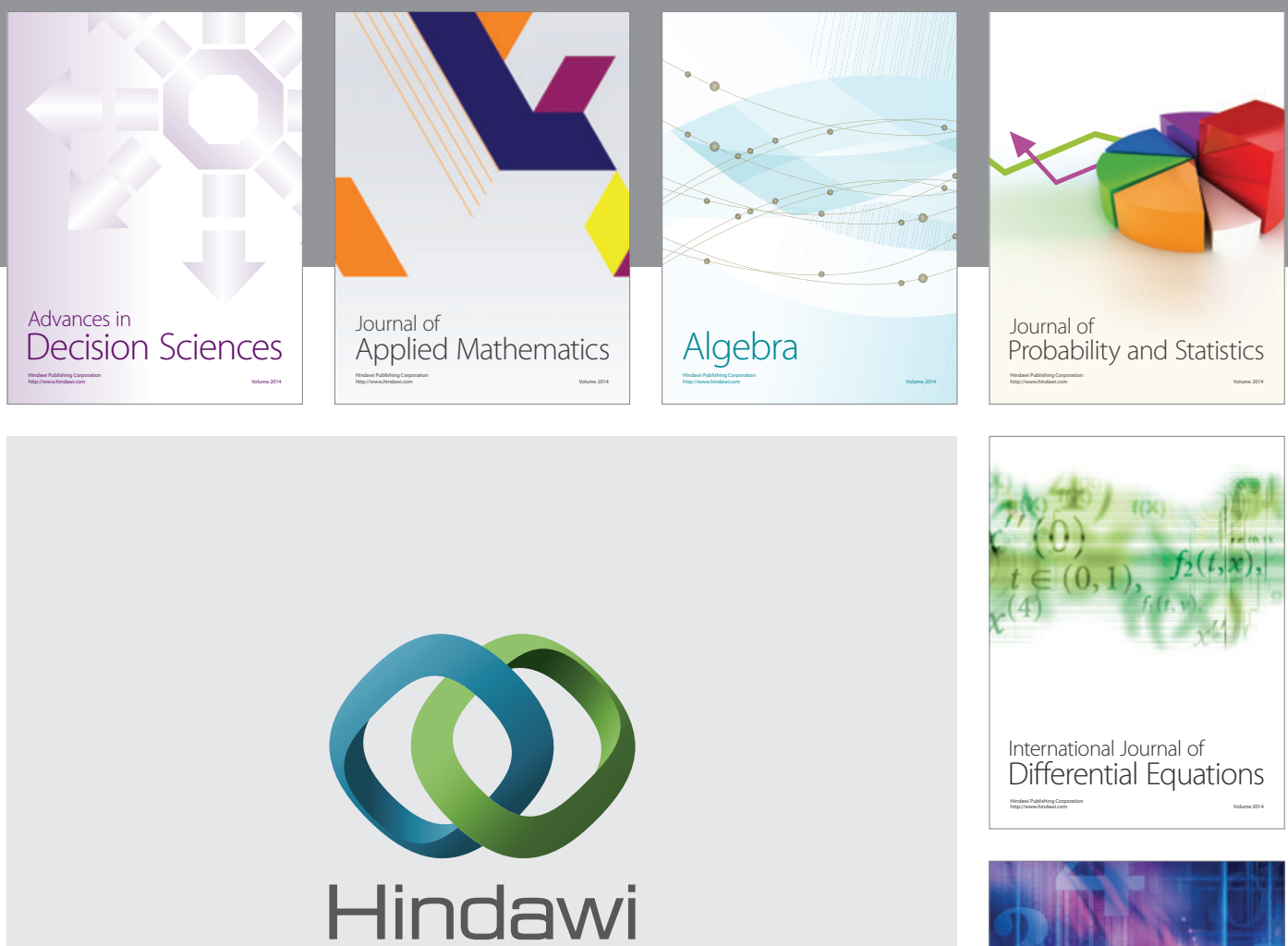

Submit your manuscripts at http://www.hindawi.com
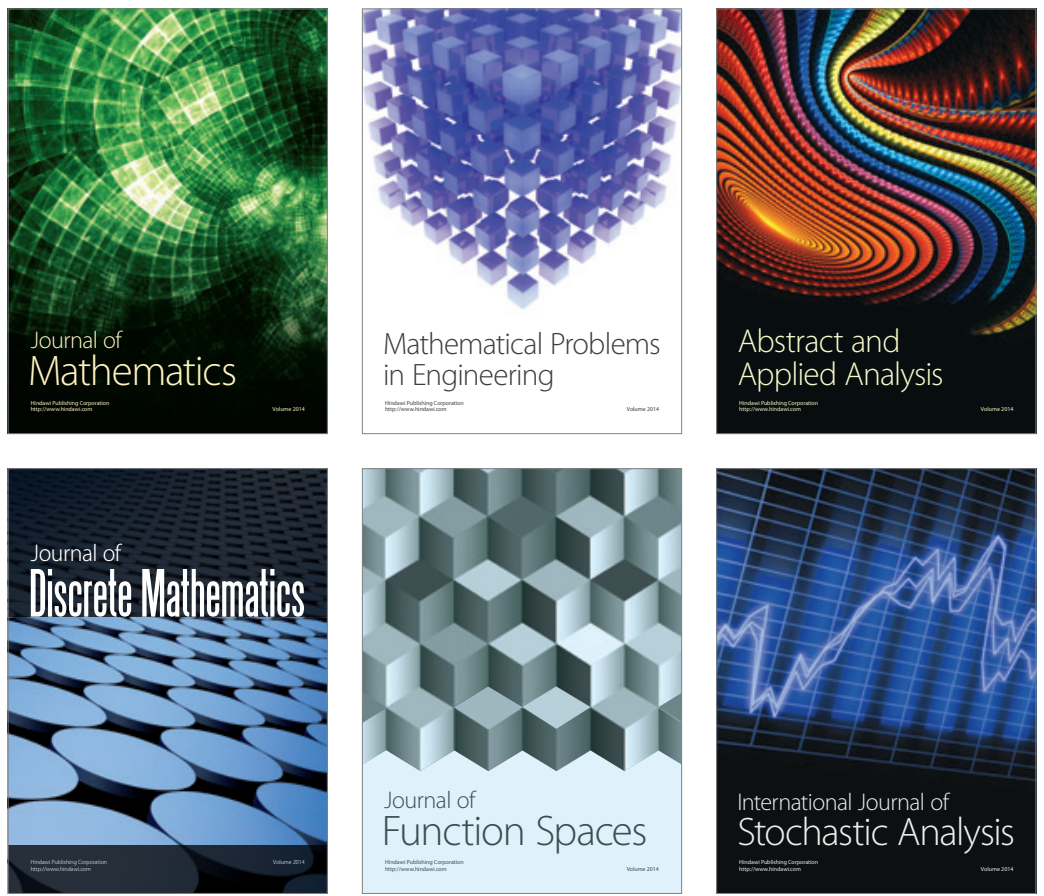

Journal of

Function Spaces

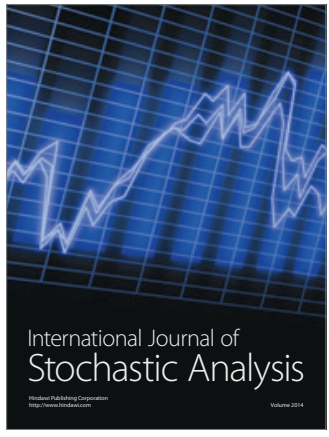

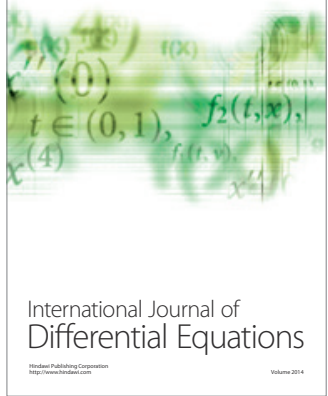
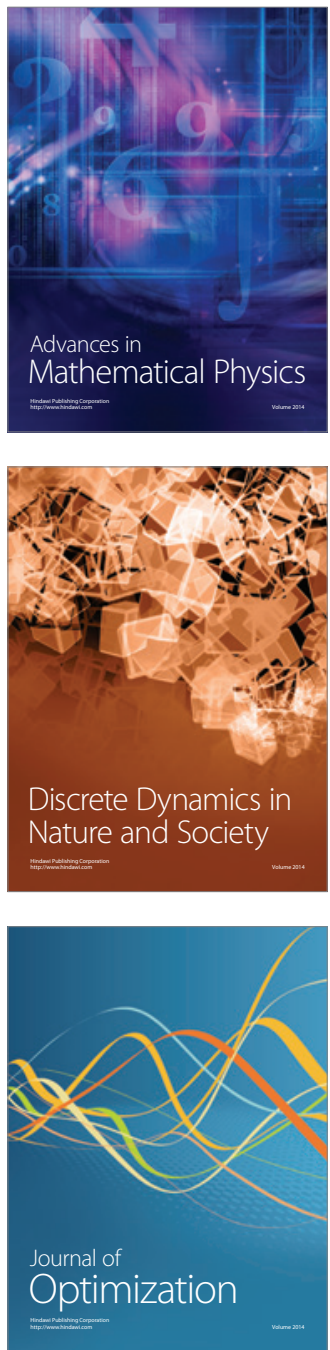